\title{
Heart failure with preserved ejection fraction in a patient with bilateral phrenic paralysis. When the biomarkers do not help the diagnosis
}

\author{
Insuficiencia cardíaca con fracción de eyección preservada en un paciente con parálisis \\ frénica bilateral. Cuando los biomarcadores no ayudan al diagnóstico
}

\author{
Ramón Maseda-Uriza ${ }^{1 *}$, Alfonso Jurado-Román², Jesús Piqueras-Flores ${ }^{1}$, Raquel Frías-García ${ }^{1}$, \\ Pedro Pérez-Díaz ${ }^{1}$ and Juan A. Requena-Ibañe ${ }^{1}$
}

${ }^{1}$ Cardiology Department, Clinical Cardiology Section; ${ }^{2}$ Cardiology Department, Hemodynamics Section. Hospital General Universitario de Ciudad Real. Ciudad Real, Spain

\section{Introduction}

Bilateral phrenic nerve paralysis is a rare cause of dyspnea, especially in the supine position and on exertion ${ }^{1}$. Many of these paralyses are related to neuropathies, and amyotrophic neuralgia is one of them. It is a rare entity of still unknown etiology described by Parsonage and Turner in 1948; in many patients, there is a history of infections, surgical interventions or vaccines $^{2}$. Heart failure $(\mathrm{HF})$ is the most commonly diagnosed heart disease; approximately half the patients have a preserved ejection fraction (HFpEF), and its main symptom is severe intolerance to exercise ${ }^{3}$. The coexistence of other comorbidities such as a chronic obstructive pulmonary disease can hinder the HFpEF diagnosis.

\section{Clinical case}

We present the case of a 45-year-old male, pastor by profession, non-smoker, occasional drinker, who attended the emergency department referring dyspnea since one month prior, which worsened with exercise and at bedtime, and that in previous week he experienced intolerance to the supine position. He referred that since one year prior, he had experienced limitation for exercising and that the current episode started after a major exertion with pain at the level of both shoulders, which he did not give importance to. On physical examination, the patient was tachypneic, with $85 \%$ oxygen saturation, HR of $102 \mathrm{bpm}$ and BP of $180 / 95 \mathrm{mmHg}$; on cardiopulmonary auscultation a decrease in vesicular murmur in both lung bases with crackling up to middle fields stood out. Initially, the patient was diagnosed with HF "not otherwise specified" and was started on oxygen mask administration and IV furosemide at $40 \mathrm{mg}$.

Chest X-ray (Figs. 1A and 1B) was reported with poor inspiration, with signs of vascular redistribution and left effusion. After consultation with the cardiology department, making a determination of BNP levels was decided, which were elevated $(235 \mathrm{pg} / \mathrm{mL})$, as well as transthoracic echocardiogram (TTE), which revealed

\section{Correspondence:}

*Ramón Maseda-Uriza

Servicio de Cardiología

Hospital General Universitario Ciudad Real

Obispo Rafael Torija, $\mathrm{s} / \mathrm{n}$

Date of reception: 02-06-2017

Date of acceptance: 10-09-2018

13005, Ciudad Real, Spain

E-mail: maseda12@ hotmail.com

DOI: 10.24875/ACME.M19000012
Available online: 09-08-2019 Arch Cardiol Mex (Eng). 2019;89(1):72-75 www.archivoscardiologia.com

2604-7063 @ 2018 Instituto Nacional de Cardiología Ignacio Chávez. Published by Permanyer México. This is an open access article under the CC BY-NC-ND license (http://creativecommons.org/licenses/by-nc-nd/4.0/). 


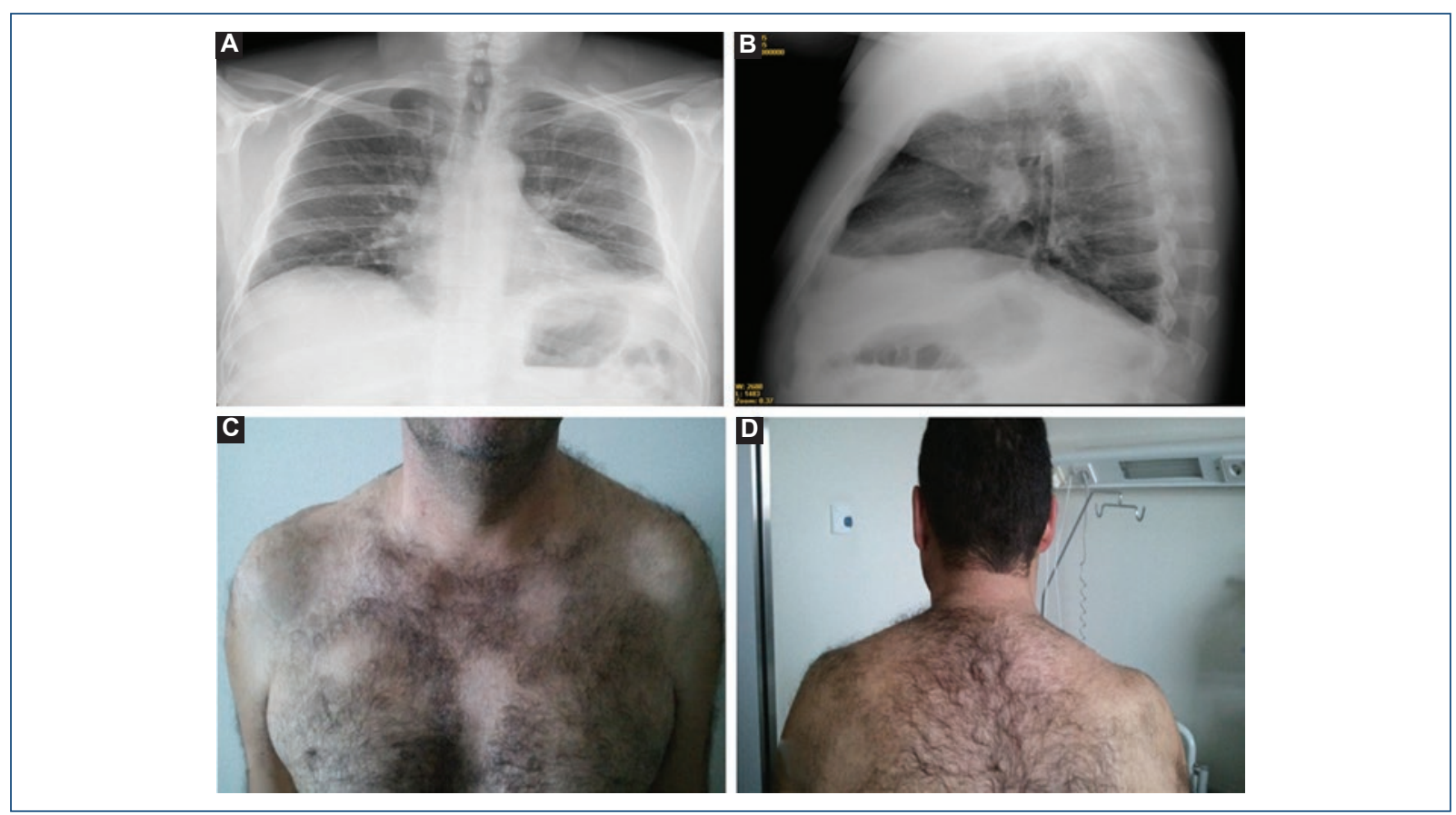

Figure 1. A and B: PA and lateral chest X-ray where diaphragmatic elevation is observed. $\mathbf{C}$ and $\mathbf{D}$ : scapular waist muscle atrophy can be observed, which is more evident on the right side.

moderate, concentric left ventricular hypertrophy (LVH), without contractility alterations, and with left ventricular fraction ejection of $58 \%$. E- and A-wave values were 0.6 and $0.2 \mathrm{~m} / \mathrm{s}$, respectively, E/A ratio was 3 , which was not modified with Valsalva maneuvers; tissue Doppler at the level of the lateral mitral ring showed an e' wave of $5 \mathrm{~cm} / \mathrm{s}$ (Figs. 2A and 2B) with a slightly dilated left atrium (LA) $\left(38 \mathrm{~mL} / \mathrm{m}^{2}\right)$, without mitral insufficiency, non-dilated right ventricle with preserved function and moderate tricuspid regurgitation that allowed calculating a pulmonary artery systolic pressure of $48 \mathrm{mmHg}$.

With an HFpEF diagnosis, hospital admission was decided in charge of the cardiology department, on treatment with IV diuretics, angiotensin converting enzyme inhibitors and beta-blockers. On the second day after admission, and despite adequate diuresis, with negative fluid balance, there was no improvement in dyspnea or orthopnea, with intolerance to the supine position and the need to sleep in the sitting position due significant desaturation, thus requiring BiPAP-mode non-invasive mechanical ventilation (NIMV) initiation.

A new chest $X$-ray showed no changes with regard to the previous one and a new TTE revealed an anomalous movement of the interventricular septum towards the left ventricle at systole and pulmonary hypertension of $55 \mathrm{mmHg}$. On physical examination, atrophy was found in the supraclavicular musculature, as well as deltoids with winged scapula (Figs. 1C and 1D) and paradoxical breathing. With suspicion of another cause for dyspnea, the pulmonology department was consulted, where spirometry showed a FEV1/FVC ratio of 57.4, FEV1 of $0.83 \mathrm{~L}(22.6 \%)$ and FVC of $1.44 \mathrm{~L}(32.6 \%)$, which was consistent with severe restriction. The neurology department performed a cervical and dorsal magnetic resonance imaging study, which was significantly limited by patient intolerance to the prone position, but there were no relevant findings. Neurophysiological testing reported acute signs of denervation in the trapezius, supraspinatus and left deltoid muscles; right spinal accessory nerve and left axillary nerve motor conduction study showed severely reduced amplitude, left spinal accessory nerve with moderately reduced motor potential, polyneuropathy and plexitis. With these data, the diagnosis of amyotrophic neuralgia with bilateral phrenic involvement was established. Diuretics and other medication for HF were discontinued and corticosteroids, group B vitamins and IV immunoglobulins administration was started, with NIMV ventilatory support being maintained. Despite treatment, the signs of denervation persisted, with poor functional recovery, and with NIMV support being required at discharge. 


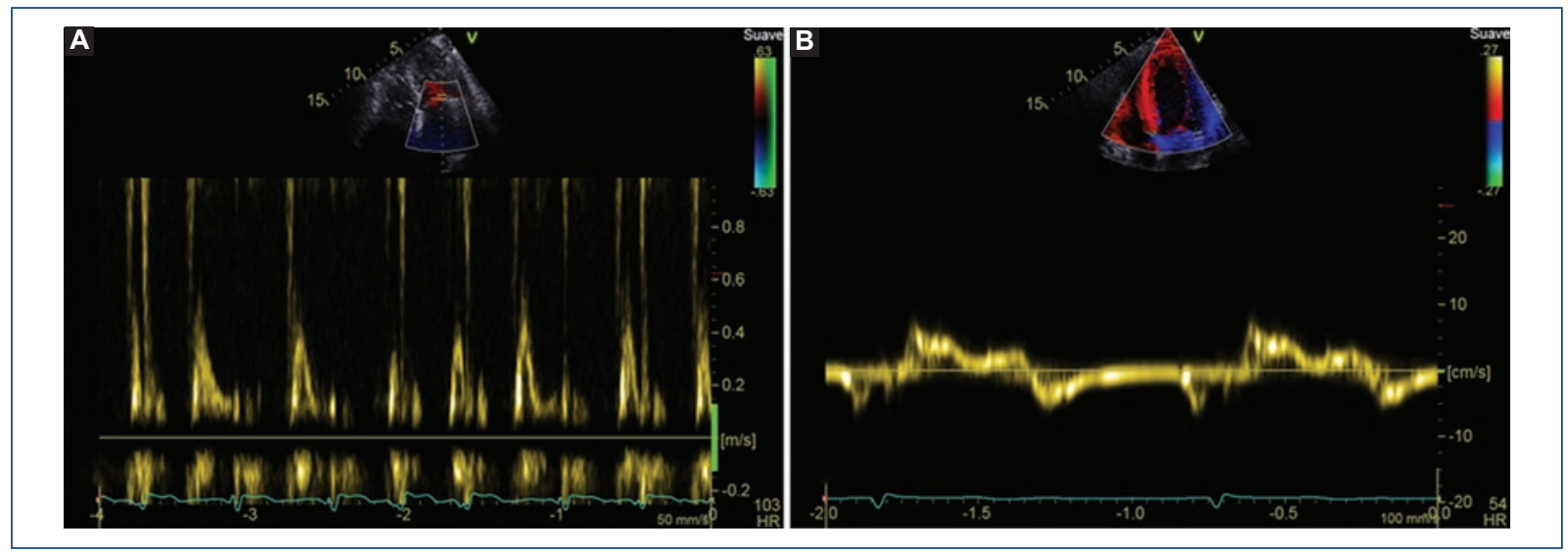

Figure 2. A: restrictive mitral filling pattern. B: lateral mitral ring TDI.

\section{Discussion}

Dyspnea in this patient was clearly related to bilateral phrenic nerve palsy in the context of amyotrophic neuralgia; however, in the echocardiographic study, there are data suggesting the HFpEF diagnosis (LA dilation, $\mathrm{LVH}$ and restrictive filling pattern $)^{3}$. The usefulness of biomarkers for dyspnea differential diagnosis is demonstrated and their elevation is one of the criteria for HFpEF diagnosis. In this case, BNP elevation was decisive for associating dyspnea with a possible cardiac cause, since it is one of the diagnostic criteria established in the latest European Society of Cardiology clinical practice guidelines (ESC 2016), if in addition we take into account that its profitability increases in patients with diagnostic uncertainty ${ }^{4}$. In our patient, there was a certain discrepancy between the intensity of dyspnea and complementary tests' findings. Amyotrophic neuralgia classic clinical presentation consists of a history of severe pain in the scapular muscles followed by progressive weakness and atrophy of cervical roots C5 and C6-innervated muscles $^{2}$. Bilateral phrenic involvement is very rare in these patients and there are very few cases described in the literature. Idiopathic unilateral paralysis is more frequent and common in men; possible causes that should be ruled out include tumors of the neck and the mediastinum and bronchogenic carcinomas ${ }^{5}$. Paradoxical breathing in patients with phrenic nerve palsy consists in abdominal wall inward movement during inspiration; this characteristic sign was overlooked on initial assessment at the emergency department and during the first hospitalization days, with more importance given to the symptom (dyspnea). It is possible that, in this case, and given the echocardiogram findings, HFpEF symptoms could have coexisted; in addition, the patient had a history of one year of intolerance to exercise. We do not consider the BNP elevation to be a false positive; what is clear, is that previous month intense dyspnea was not of cardiac etiology. Reviewing amyotrophic neuralgia with bilateral phrenic involvement published cases, most of them have been previously admitted to the pulmonology department and are diagnosed with restrictive alterations based on functional tests' results. Vital capacity worsening in the supine position due to abdominal viscera displacement towards the chest is characteristic in these patients. For diagnosis, the use of fluoroscopy is useful, which shows the diaphragmatic paradoxical movement; in the neurophysiological study, the slowing down of conduction velocities is characteristic ${ }^{5}$. For the treatment of amyotrophic neuralgia, corticosteroids or IV immunoglobulins have been used with different success rates. In our case, there was no improvement despite the use of both options. For the management of phrenic nerve paralysis, BiPAP- or CPAP-mode NIMV is used; if there is not improvement, there are surgical options, such as diaphragmatic plication in case of unilateral paralysis or implantation of a phrenic pacemaker ${ }^{6}$.

\section{Conclusions}

Although in current medicine the use of biomarkers is prevailing to aid dyspnea differential diagnosis, it is important not to forget that a good physical examination can guide it and should not be replaced. HFpEF can coexist with other pathologies that mask its diagnosis 
and therefore it is important for dyspnea differential diagnosis to be taken into account.

\section{Conflicts of interest}

The authors declare not having any conflicts of interest or having received funding.

\section{Ethical disclosures}

Protection of people and animals The authors declare that no experiments have been conducted on humans or animals for this investigation.

Confidentiality of data The authors declare having followed the protocols of their work center on the publication of patient data.
Right to privacy and informed consent The authors declare that no patient data appear in this article.

\section{References}

1. Ikegami G, Abe T, Akasaka K, Kouyama A, Souma R, Matsuo T, et al Bilateral phrenic nerve paralysis manifested by orthopnea for 6 months in a patient with neuralgic amyotrophy. Intern Med. 2009;48(24):2123-7.

2. Lahrmann H, Grisold W, Authier FJ, Zifko UA. Neuralgic amyotrophy with phrenic nerve involvement. Muscle Nerve. 1999;22(4):437-42.

3. Montero D, Flammer AJ. Exercise intolerance in heart failure with preserved ejection fraction: time to scrutinize diuretic therapy ? Eur J Heart Fail. 2017;19(8):971-3.

4. Steinhart B, Thorpe KE, Bayoumi AM, Moe G, Januzzi JL Jr, Mazer CD. Improving the diagnosis of acute heart failure using a validated prediction model. J Am Coll Cardiol. 200913;54(16):1515-21.

5. Gayan-Ramirez G, Gosselin N, Troosters T, Bruyninckx F, Gosselink R, Decramer M. Functional recovery of diaphragm paralysis: a long-term follow-up study. Respir Med. 2008;102:690-8.

6. Rafiq A, ljaz M, Tariq H, Vakde T, Duncalf R. Failing phrenics: an obscure cause of exertional dyspnea: Case report and literature review. Medicine (Baltimore). 2016;95(29):42-63. 